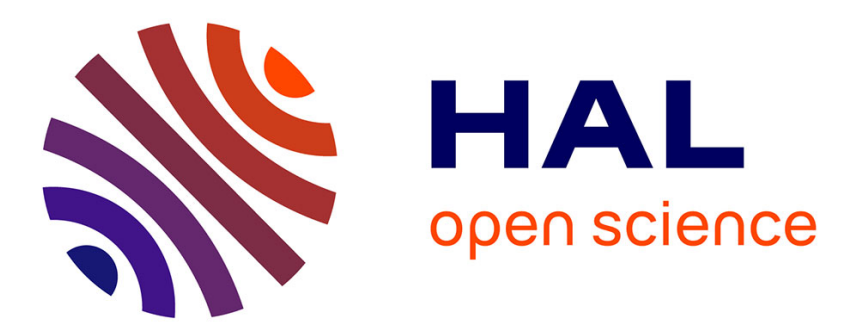

\title{
A model for the metallic resistivity of TTF-TCNQ \\ D. Jérome
}

\section{To cite this version:}

D. Jérome. A model for the metallic resistivity of TTF-TCNQ. Journal de Physique Lettres, 1977, 38 (24), pp.489-494. 10.1051/jphyslet:019770038024048900 . jpa-00231427

\section{HAL Id: jpa-00231427 https://hal.science/jpa-00231427}

Submitted on 1 Jan 1977

HAL is a multi-disciplinary open access archive for the deposit and dissemination of scientific research documents, whether they are published or not. The documents may come from teaching and research institutions in France or abroad, or from public or private research centers.
L'archive ouverte pluridisciplinaire HAL, est destinée au dépôt et à la diffusion de documents scientifiques de niveau recherche, publiés ou non, émanant des établissements d'enseignement et de recherche français ou étrangers, des laboratoires publics ou privés. 


\title{
A MODEL FOR THE METALLIC RESISTIVITY OF TTF-TCNQ (*)
}

\author{
D. JÉROME \\ Laboratoire de Physique des Solides (**) \\ Université Paris-Sud, 91405 Orsay, France
}

(Reçu le 20 octobre 1977, accepté le 22 novembre 1977)

\begin{abstract}
Résumé. - Nous proposons dans cette lettre un nouveau mécanisme de diffusion pour la résistivité, basé sur l'existence de collisions entre électrons et modes collectifs (du type ondes de spins). Ce mécanisme est d'une efficacité toute particulière dans TTF-TCNQ compte tenu de la proximité de l'ordre magnétique $\left(U / 4 t_{\|} \sim 1\right)$. Il conduit à une loi de dépendance de la résistivité à axe $b$ constant en fonction de la température qui est quasi linéaire et à une connexion entre propriétés de conduction et propriétés magnétiques dans TTF-TCNQ ainsi que dans les composés dérivés.
\end{abstract}

\begin{abstract}
We propose a new scattering mechanism for the resistivity based on electron lowlying collective mode (spin wave like) collisions. This scattering is particularly efficient in TTFTCNQ as magnetic ordering is nearly achieved in this material under ambient conditions $\left(U / 4 t_{\|} \approx 1\right)$. Also it provides a theoretical quasi-linear temperature dependence at constant $b$-axis together with the essential connection noticed between conductivity and magnetism in TTF-TCNQ and all derivative compounds.
\end{abstract}

1. Introduction. - No generally accepted picture has yet emerged for the electronic properties of the charge transfer salt TTF-TCNQ in the metallic region above $60 \mathrm{~K}$. There exist several different and sometimes mutually exclusive points of view [1,2].

i) Heeger [3] and co-workers have attributed the anomalous optical properties, the strong temperature dependence of the static susceptibility (a factor 2 decrease from 300 to $100 \mathrm{~K}$ ) and the temperature dependence of the $\mathrm{C}^{13} \mathrm{Knight}$ shift to a pseudo gap in the quasi-particle density of states brought about by strong 1-D charge fluctuations which exist between a high mean field Peierls transition temperature and a low 3-D ordering temperature. The DC conductivity was ascribed to a collective electron-phonon effect.

In contrast Thomas et al. [4] consider that there is no need to invoke collective current carrying excitations to account for the observed conductivity. The nearly quadratic temperature dependence of the resistivity in many organic conductors has been attributed to electron-electron scattering by Seiden and Cabib [5].

ii) More recently, Conwell [6] has proposed an explanation of the resistivity by scattering of the carriers against optical molecular phonons, whereas

(*) This work has been performed in part with a D.G.R.S.T. contract $\mathrm{n}^{\circ}$ 75-7-0820.

(**) Laboratoire associé au C.N.R.S.
Gutfreund and Weger [7] have suggested that the scattering occurs against the rigid rotations of the molecules (librations).

The work of Rashba et al. [8] and Madhukar and Cohen [9] on thermally induced localization might be appropriate for TTF-TCNQ, since at room temperature the electronic mean free path as deduced from the conductivity is of the order of the $b$-axis lattice spacing. This indicates that near room temperature and above conductivity should probably be treated as diffusive. Consequently the conductivity would be limited by hopping between localized states rather than by scattering from one extended state to another.

iii) Another school (Torrance et al. [10], Jérome et al. [11]) believes that the on-site Coulomb repulsion $U$ might play an important role in metallic charge transfer salts. The possibility of $U$ being about the same size as the electron bandwidth $4 t_{\|}$has been supported by a wide spectrum of data obtained at Orsay [12], for which high pressure technique has proved to be indispensable, in particular the pressure dependence of the susceptibility [13]. In attempting to clarify the situation a great deal of attention has been paid to the measured temperature dependence of Fermi level properties in the region 60-300 especially the conductivity and magnetic susceptibility.

In particular it has recently been shown by Cooper and Jérome [14] that the temperature dependence 
of the resistivity can be very misleading due to the combined effects of the large $b$-axis thermal expansion and the fact that all Fermi level properties are anomalously pressure (i.e. volume) dependent [15]. The corrected temperature dependence of the resistivity (at constant $b$-axis) has been derived in reference [14].

As a result the intrinsic behaviour (constant $b$-axis) of the resistivity is quasi-linear in temperature instead of the $T^{2.3}$ temperature dependence at constant pressure [16]

In section 2 we shall discuss the constant $b$-axis resistivity in connection with various resistivity models. A new model for the resistivity, based on the scattering of charge carriers off spin collective modes is proposed in section 3. This model supports the intermediate $U$ picture proposed for TTFTCNQ [11]. Finally in section 4 we show how this new model establishes the connection between conductivity and magnetism in TTF-TCNQ and all derivative compounds.

2. Constant $b$-axis resistivity : consequences. The intrinsic temperature dependence of the constant $b$-axis resistivity is shown on figure 1 following a procedure similar to the of reference [14].

Other properties also show substantial changes under pressure : the transverse conductivity [17], the spin susceptibility [13], the NMR relaxation rate [18] and the ESR linewidth [19]. Therefore we feel that the actual constant b-axis $T$ dependence of these quantities should be reduced by this correction. The particular case of the susceptibility will be the subject of a separate publication [20]. Recent experiments of elastoresistance on TTF-TCNQ [21] have shown that the influence of a strain on the $b$-axis conductivity is about 10 times larger when the stress is applied along the $b$ direction than along a transverse direction. Therefore we feel justified in considering the $b$-axis conductivity to be sensitive to deformations of only the $b$-axis when a single crystal is put under hydrostatic pressure. Taking into account the strong volume dependence and the $T$-like temperature dependence of the resistivity at constant $b$-axis

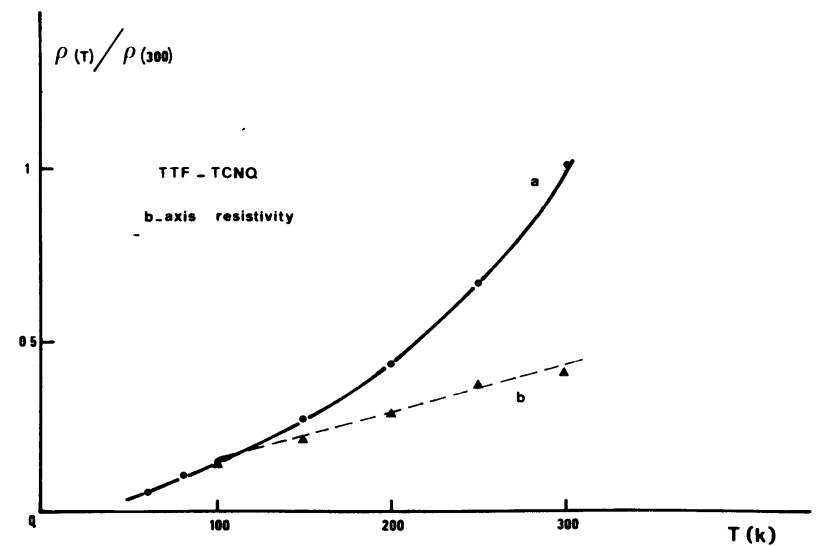

FIG. 1. - $a$ ) Experimental $b$-axis resistivity of TTF-TCNQ between 60 and $300 \mathrm{~K} ; b$ ) Corrected to constant $b$-axis. it appears that the resistivity mechanisms based on scattering against optical molecular phonons [6], or against librations [7] cannot explain the constant volume data. Scattering by librations gives a $T^{2}$ temperature dependence at constant volume instead of the linear dependence found experimentally. However the effect of thermal expansion is likely to increase the exponent of the power law above 2 , since $\omega_{\text {lib }}$ must drop as the volume increases. Any process based on scattering against acoustical phonons seems to have to be discarded as well. Such processes could produce a linear temperature dependence at $T>\theta_{\mathrm{D}},(\approx 100 \mathrm{~K})$. However, the pressure dependence can be related to the pressure dependence of the electron-phonon coupling constant, $\lambda$,

$$
(\partial \ln \rho / \partial P \sim \partial \ln \lambda / \partial P) .
$$

High pressure studies of the Peierls transition have given

$$
\left|\frac{\partial \ln \lambda}{\partial P}\right| \sim 1 \% / \operatorname{kbar} \quad[17]
$$

in contrast to observed

$$
\frac{\partial \ln \sigma}{\partial P}=28 \% / \mathrm{kbar}
$$

in TTF-TCNQ. The value of the electron phonon coupling constant has been derived from the Peierls temperature [17] namely $\lambda \approx 0.2$. This value of $\lambda$, together with Hopfield's relation [22] and the plasma frequency $\omega_{\mathbf{P}}=1.3 \mathrm{eV}$ would lead for TTF-TCNQ to a value of the room temperature resistivity which is roughly 10 times lower than what is observed. As far as phonon-localization is concerned we believe that this theory could very well account for the behaviour of the conductivity in compounds where static disorder is important such as systems like $\mathrm{D}^{+}\left(\mathrm{TCNQ}_{2}\right)^{-}$[23] where $\mathrm{D}$ is a non-symmetric donor molecule, but not for the case of weakly disordered systems such as TTF-TCNQ. The pressure (temperature) dependence of $\sigma=n e^{2} \tau / m^{*}$ can be decomposed into a pressure (temperature) dependence of $n / m^{*}$ and $\tau$. The ratio $n / m^{*}$ can be directly related to the plasma frequency

$$
\omega_{\mathrm{P}}^{2}=\frac{4 \pi n e^{2}}{m^{*}}
$$

Let us first look at the pressure dependence at $300 \mathrm{~K}$.

The density of carriers available for conduction in the $1-\mathrm{D}$ metal at $300 \mathrm{~K}$ and $1 / m^{*}$ is proportional to the charge transfer $\rho$ and the bandwidth $4 t_{\|}$. Therefore, from eq. (1)

$$
\frac{2 \partial \ln \omega_{\mathrm{P}}}{\partial P}=\frac{\partial \ln \rho}{\partial P}+\frac{\partial \ln t_{\|}}{\partial P} .
$$


A volume dependence of $k_{\mathrm{F}} / b^{*}$ has been noticed in $\mathrm{X}$-ray scattering experiments from the so called $4 k_{\mathrm{F}}$ reflections $[24,25]$. The position of the $4 k_{\mathrm{F}}$ scattering shifts towards larger values of $\rho$ at low temperatures.

Assuming that $k_{\mathrm{F}}$ is sensitive to the $b$-axis only and equivalence between high pressure and thermal contraction we derive :

$$
\frac{\partial \ln k_{\mathrm{F}}}{\partial P} \approx 1 \% / \operatorname{kbar}[26] \text { and } \frac{\partial \ln \rho}{\partial \ln b} \approx-2 .
$$

The slight increase of $\omega_{\mathbf{P}}$ noticed under pressure in optical reflectance experiments [27]

$$
\frac{2 \partial \ln \omega_{\mathrm{P}}}{\partial P} \approx 2.5 \% / \mathrm{kbar}
$$

is consistant with the pressure dependences of charge transfer and bandwidth (see equation 2)

$$
\frac{\partial \ln \rho}{\partial \ln b} \sim-2, \quad \frac{\partial \ln t_{\|}}{\partial \ln b} \sim-4
$$

to within the accuracy of the Drude fits.

The interpretation of temperature dependences is not straight-forward since the transverse conductivity undergoes a diffusive to coherent transition at low temperature $[12,18]$. Although this transition is not actually observed in TTF-TCNQ where a lattice distortion occurs first at $53 \mathrm{~K}$ in cooling, pretransitional effects have been observed, especially in the Hall constant below $\approx 150 \mathrm{~K}$ [28]. For this reason temperature dependences of $n, m^{*}$ and therefore $\omega_{\mathrm{p}}$ are not simply related to the temperature dependences of $k_{\mathrm{F}}$ and $t_{\|}$.

However, small temperature dependences of $\omega_{\mathbf{P}}$ have been observed in TTF-TCNQ between $300 \mathrm{~K}$ and $80 \mathrm{~K}$,

$$
\frac{\Delta \omega_{\mathbf{P}}}{\omega_{\mathbf{P}}} \lesssim+3 \%
$$

according to various authors [29].

It becomes now clear that the main temperature and pressure dependence of $\sigma$ are provided by $\tau$.

3. A model for the resistivity. - The possibility that the on-site Coulomb repulsion $U$ may be of the same order as the electron bandwidth $4 t_{\|}$is suggested by the enhancement and by the temperature dependence of the spin susceptibility, by the enhancement of the $T_{1}$ proton relaxation and by very large pressure dependences of these two quantities. Obviously, the $T_{1}$ and $\chi$ pressure dependences cannot be explained solely by the band broadening occuring under pressure, since in TTF-TCNQ $[13,18]$.

$$
\frac{\partial \ln \chi}{\partial \ln b}=20 \text { and } \frac{\partial \ln T_{1}^{-1}}{\partial \ln b}=50
$$

whereas

$$
\frac{\partial \ln t_{\|}}{\partial \ln b}=-4 .
$$

The possibility that TTF-TCNQ may exhibit some features characteristic of the large $U$ limit, for example a soft phonon at $4 k_{\mathrm{F}}$ and spin-wave modes at $2 k_{\mathrm{F}}$ has been proposed by Torrance $[10,30]$ following the Hubbard model calculation of the excitation spectrum of Coll [31]. Emery pointed out also the effect of the correlations as the origin of the $4 k_{\mathrm{F}}$ scattering [32].

In addition, Torrance [10] has proposed a description for TTF-TCNQ based on antiferromagnetic Wigner waves and Hubbard [33], after calculation of the various Coulomb interactions, explain the $\mathrm{CDW}$ in terms of Wigner localization of strongly correlated electrons.

However TTF-TCNQ exhibits may specific features that a $U=0$ (or small $U$ ) model would predict. The most specular being the existence of Bragg satellites $\pm 2 k_{\mathrm{F}}$ away from the main Bragg spots on X-ray diffraction patterns and a soft phonon mode at $2 k_{\mathrm{F}}$. It should also be kept in mind that the method of approximation used in the interpretation of $T_{1}^{-1}$ in TTF-TCNQ [18] is in essence a small $U$ approximation; although the final result we arrived at was $U \sim 4 t_{\|}$. It is for all these reasons that we took the intermediate $U$ picture for TTF-TCNQ. So far no appropriate theory exists, for the description of the intermediate coupling. In particular it is probably not even right to speak in terms of Fermi surfaces in a strongly correlated system such as TTF-TCNQ. We shall now propose an approach to the problem of the resistivity which is obviously approximate, but which contains much of the physics that an exact theory should include. A year ago [11] we suggested a model for the intra chain electron scattering based on the existence of scattering of charge carriers by spin fluctuations. However the agreement between the erroneous $T^{2}$ temperature dependence (only true in $3 \mathrm{D}$ ) and experiment was fortuitous since the effect of thermal expansion was not taken into account.

In 1-D conductors the only processes leading to resistivity involve a momentum transfer of $\approx 2 k_{\mathrm{F}}$ (back scattering). As long as a single band is concerned electron-electron scattering leads to a $1 / \tau \sim T$ law only for a half-filled band (Umklapp processes). Quasi-elastic collisions between an incident electron at the Fermi level, with momentum $\mathbf{k}_{1}=\mathbf{k}_{\mathbf{F}}$ and a target electron of momentum $\mathbf{k}_{2}$ must satisfy the momentum conservation law :

$$
\mathbf{k}_{1}+\mathbf{k}_{2}=\mathbf{k}_{1}^{\prime}+\mathbf{k}_{2}^{\prime}+4 \mathbf{k}_{\mathrm{F}}
$$

( $4 \mathbf{k}_{\mathrm{F}}=$ Reciprocal lattice vector).

Since the collision is quasi elastic $\mathbf{k}_{2}=\mathbf{k}_{\mathrm{F}}$ (imposing a Fermi factor $T / T_{\mathrm{F}}$ in $1 / \tau$ ) 
In 1-D, the final states $\mathbf{k}_{1}^{\prime}=\mathbf{k}_{2}^{\prime}=-\mathbf{k}_{\mathrm{F}}$ are entirely defined by the momentum relation (1) in contrast to 3-D where the number of allowed final states introduces another $T / T_{\mathrm{F}}$ factor.

Therefore, electron-electron scattering varying linearly with $T$ is a property of 1-D Fermi surfaces (as already mentionned by Gorkov and Dzialoshinskii [34]).

Since TTF-TCNQ is not a half-filled band charge transfer salt, intraband weakly correlated e-e scatterings cannot explain the resistivity.

One may also consider e-e scattering between carriers belonging to two different bands (interband process). Such a mechanism is however very unlikely since under ambient conditions the electrons are confined to a given chain for a duration about 1000 times larger than the scattering time [18].

The excitation spectrum of the 1-D Hubbard model has been calculated by Coll for a band filling less than unity [31]. The existence of a non zero on-site Coulomb repulsion shifts the softening of the particle-hole excitations from the $2 k_{\mathrm{F}}$ value at $U=0$ to $4 k_{\mathrm{F}}$. Moreover it gives rise to low lying collective excitations associated with incommensurate antiferromagnetism. The exact solution for the excitations of a 1-D correlated electron gas has not been produced yet. But the excitation spectrum can be expected to show softening of the particle-hole excitations at $4 k_{\mathrm{F}}$ and at $2 k_{\mathrm{F}}$, together with some low lying collective modes similar to the spin waves of Coll's model.

Tentatively one can describe the excitation spectrum of the intermediate $U$ situation as the superposition of $U=0$ and $U>0$ spectra, figure 2 . In following this precedure it is implicitly assumed that

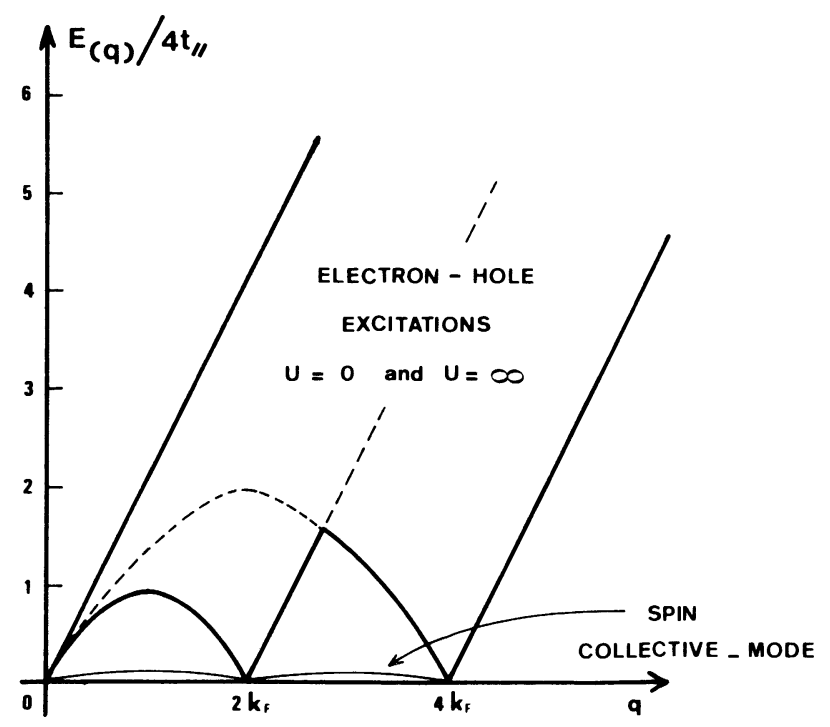

FIG. 2. - A schematic excitations spectrum expected for the intermediate $U$ situation. It includes the free-electron spectrum with a soft mode at $2 k_{\mathrm{F}}$ and the correlated spectrum derived from Coll's calculation [31] with a soft mode at $4 k_{\mathrm{F}}$ and low-lying collective modes for $\left(U / 4 t_{\|} \sim 1\right)$. coupling between single particle and collective modes is neglected.

The scattering of an electron at $k_{\mathrm{F}}$ off the collective mode at $2 k_{\mathrm{F}}$ transfers a momentum $2 k_{\mathrm{F}}$ from the charge carrier system to the collective mode. Then, momentum $2 k_{\mathrm{F}}$ decays easily to the lattice through a collective mode-lattice interaction. A spin flip of the electron occurs during the scattering process [35].

Quite generally the scattering time becomes

$$
1 / \tau \propto \frac{T}{T_{J}}\left(S\left(2 k_{\mathrm{F}}\right)\right)^{2}
$$

where $S(q)$ is a scattering amplitude, expressing the amount of order in the spin system, and $T_{J}$ is the characteristic temperature of the collective mode excitations $\left(k T_{J} \approx J\right)$.

It has been shown [36] that the scattering amplitudes increase logarithmically with falling temperature for electrons with momenta close to $\pm k_{\mathrm{F}}$. The scattering amplitude can be derived in the Hubbard model with $U>0$, using the so called parquet diagram approximation and develops a pole at low temperatures. Actually an exact 1-D treatment of the scattering amplitude should depress the pole to zero temperature, because of the large fluctuations. At high temperature however, when short range order has not been established it is reasonable to approximate the exact scattering amplitude by its meanfield value in the Hubbard model

$$
S(q) \sim \frac{1}{1-U n\left(E_{\mathrm{F}}\right) F(q, T)} .
$$

The electron-collective mode scattering time becomes

$$
1 / \tau \sim \frac{T}{T_{J}}\left(\frac{1}{1-U n\left(E_{\mathrm{F}}\right) F\left(2 k_{\mathrm{F}}, T\right)}\right)^{2}
$$

provided that $T / T_{J} \lesssim 1$

and the volume dependence of $1 / \tau$ reads

$$
\frac{\partial \ln (1 / \tau)}{\partial \ln b}=-\frac{\partial \ln t_{\|}}{\partial \ln b} \frac{2 \operatorname{Un}\left(E_{\mathrm{F}}\right) F\left(2 k_{\mathrm{F}}, T\right)}{1-\operatorname{Un}\left(E_{\mathrm{F}}\right) F\left(2 k_{\mathrm{F}}, T\right)} .
$$

It is clear from eq. (4) that the volume dependence of the conductivity depends critically on the value of $U n\left(E_{\mathrm{F}}\right)$.

Using

$$
\frac{\partial \ln 1 / \tau}{\partial \ln b}=60
$$

and $F\left(2 k_{\mathrm{F}}, 300\right)=1.36$ we derive from (4)

in TTF-TCNQ at $300 \mathrm{~K}$.

$$
\operatorname{Un}\left(E_{\mathrm{F}}\right)=0.64
$$

From eq. (3) we see at once that the enhancement factor for the resistivity $\left[1-U n\left(E_{\mathrm{F}}\right) F\left(2 k_{\mathrm{F}}, 300\right)\right]^{-2}$ is $\sim 100$ at room temperature. So far we have consi- 
dered only charge fluctuations at $2 k_{\mathrm{F}}$ scattered by spinwaves at $2 k_{\mathrm{F}}$ and consequently a value of the scattering rate has been derived. However, other pictures could have been used, for instance charge fluctuations at $4 k_{\mathrm{F}}$ scattered by a spin wave at $4 k_{\mathrm{F}}$ or 2 spin waves at $2 k_{\mathrm{F}}$. But we do not believe that these scattering channels are likely to give scattering rates significantly larger than those proposed above. We wish to reemphasize that a fully satisfactory model of the resistivity of TTF-TCNQ requires a correct treatment of the 1-D $\left(U \approx 4 t_{\|}\right)$electron gas, which is beyond the scope of this paper.

4. Discussion of the model and conclusion. - A consequence of the magnetic model for the resistivity is its extreme sensibility to the $b$-axis lattice constant. A $100 \%$ variation of the enhancement factor, (the difference between constant pressure and constant $b$-axis) (see Fig. 1 ) is afforded by only a $5 \%$ change of $\operatorname{Un}\left(E_{\mathrm{F}}\right) F\left(2 k_{\mathrm{F}}, T\right)$, corresponding to a band broadening

$$
\frac{\Delta t_{\|}}{t_{\|}} \approx 12 \%
$$

\section{between 300 and $80 \mathrm{~K}$.}

The derived value of the band broadening is in good agreement with the pressure dependence of the plasma frequency, section 2 . In this article we have not made any distinction between the 2 different chains. This approach seems justified since $\operatorname{Un}\left(E_{\mathrm{F}}\right)$ derived from the enhancement of susceptibility, NMR and the pressure dependence of $\chi$ is $\sim 0.7$ in very good agreement with 0.66 derived from resistivity in this work.

By analogy with spin disorder resistivity in nearly magnetic metals [37] we can rewrite the magnetic resistivity as :

$$
\rho=\rho_{\infty} \frac{T}{T_{J}}\left(S\left(2 k_{\mathrm{F}}\right)\right)^{2}
$$

with

$$
\rho_{\infty} \sim \frac{m^{*}}{n e^{2} \tau_{\mathrm{F}}}\left(G n\left(E_{\mathrm{F}}\right)\right)^{2}
$$

apart from factors of the order of $\pi$ or 2 where $E_{\mathrm{F}}=\hbar / \tau_{\mathrm{F}}$, with the assumption that the ratio of conducting to magnetic electrons is equal to unity. In eq. (6), $G$ is the coupling between collective mode and single particle excitations.

With the usual set of parameters for TTF-TCNQ $T_{\mathrm{F}}=1000 \mathrm{~K}, T_{\mathrm{J}} \sim 300 \mathrm{~K}, \omega_{\mathrm{P}}=1.38 \mathrm{eV}$,

$$
\left(\frac{m^{*}}{n e^{2}}=\frac{4 \pi}{\omega_{\mathrm{P}}}\right), \quad \sigma(300)=500(\Omega \mathrm{cm})^{-1},
$$

we derive from (5) and (6) $G n\left(E_{\mathrm{F}}\right) \sim 0.2$. This means that the coupling between single particle and collective mode excitations around $2 k_{\mathrm{F}}$ is quite significant [38]. Otherwise no resistivity is expected from our model. But this fact indicates clearly that the whole picture (in particular figure 2) is not self-consistent and that an improvement of the theory is needed in a proper treatment of the mode coupling.

The strongly correlated electron system exhibits three characteristic energies.

i) the exchange energy, $J \sim 300 \mathrm{~K}$,

ii) the coupling between collective mode and single particle excitations, $G \sim 1300 \mathrm{~K}$,

iii) the on-site Coulomb repulsion, $U \sim 4000 \mathrm{~K}$.

Actually in Coll's model [31] $J$ and $U$ are related since for $\rho=0.59 \mathrm{el} / \mathrm{molecule}, J=1.3 t_{\|}^{2} / U$, leading again to $U / 4 t_{\|} \approx 1$. The finding of a quasi-linear law for the constant $b$-axis temperature dependence of the resistivity is somewhat puzzling (Fig. 1), when eq. (3) is taken into account. The scattering amplitude at constant $b$-axis depends logarithmically upon temperature in the mean-field approximation.

However a proper one dimensional derivation of $S(q)$ should provide a divergence only at zero temperature and a temperature dependence presumably much weaker than that given by the mean-field approximation, justifying therefore the data of figure 1 . Actually, the fact that the scattering amplitude is not diverging at low temperature indicates the limit of validity of the mean-field approach. This approximation becomes probably inadequate for the description of 1-D spin fluctuations in TTF-TCNQ below, say, $150 \mathrm{~K}$.

Another problem is the behaviour of the resistivity in the vicinity of the resistivity minimum $(60<T<100 \mathrm{~K})$.

Between 60 and $100 \mathrm{~K}$ the thermal expansion along the $b$-axis is at most $\approx 0.26 \%$ [39], corresponding to a pressure effect of $\approx 500$ bars [40].

From our early study of TTF-TCNQ [15] this pressure effect would result in a $\approx 3 \%$ decrease of the resistivity for a constant $b$-axis behaviour. Consequently, we may say that the thermal expansion correction considered above is negligible below $80 \mathrm{~K}$ and that constant pressure or constant volume resistivity are similar. It is thus apparent that the quasi-linear temperature dependence observed between 100 and $300 \mathrm{~K}$ does not extend down to $60 \mathrm{~K}$. The temperature dependence of the resistivity below $80 \mathrm{~K}$ follows a power law $\rho \approx T^{\lambda}$ with $\lambda>2$ for samples exhibiting resistivity ratios $\rho(300) / \rho(60)$ larger than 10 approximately.

Although we are aware that this temperature dependence below $80 \mathrm{~K}$ may be of great importance as far as the properties of TTF-TCNQ are concerned we believe that new and better experiments are needed in order to give a definite conclusion.

In summary, we have proposed in this article a 
resistivity mechanism for TTF-TCNQ which explains the large volume dependence of the scattering time. The model is based on the scattering of particles against spin wave-like collective modes. This new mechanism satisfies the requirements of the particular resistivity properties of TTF-TCNQ, namely an extreme sensitivity to the bandwidth $4 t_{\|}$and a quasilinear temperature dependence at constant $b$-axis. Moreover, this model has introduced the essential correlation existing between conductivity and magne- tism in TTF-TCNQ and also among derivative compounds [20].

Acknowledgments. - I am particularly grateful to my collaborators at Orsay and Zagreb, J. R. Cooper and R. H. Friend with whom I had several fruitful discussions, and who made many clarifying remarks. I wish to acknowledge numerous useful discussions with S. Barisié, L. Caron, J. Friedel, G. Grüner and M. Weger.

\section{References}

For an up to date presentation of various points of view on Organic Conductors see references [1] and [2].

[1] Chemistry and Physics of One-Dimensional Metals, H. J. Keller, Editor (Plenum Press) 1977 and

[2] Organic Conductors and Semiconductors, Proceeding $\mathrm{n}^{0} 65$ (Springer) 1977.

[3] Heeger, A. J., in reference [1].

[4] Thomas (G. A.) et al. (Collective paper with 30 co-authors) Phys. Rev. B 13 (1976) 5105.

[5] Seiden, P. E. and CabiB, D., Phys. Rev. B 13 (1976) 1846.

[6] Conwell, E. M., Phys. Rev. Lett. 39 (1977) 777.

[7] Gutfreund, H. and Weger, M., Phys. Rev. B 16 (1977) 1753.

[8] Rashba, E. I., Gogolin, A. A. and Mel'nikov, V. I. in reference [2].

[9] Madhukar, A. and Cohen, M. H., Phys. Rev. Lett. 38 (1977) 85.

[10] TorranCE, J. B., in reference [1].

[11] JÉrome, D. and Giral, L., in reference [2].

[12] JÉROME, D. and WEGER, M., in reference [1].

[13] Jérome, D., Soda, G., CoOper, J. R., Fabre, J. M. and GIRAL, L., Solid State Commun. 22 (1977) 319.

[14] CoOper, J. R. and Jérome, D., submitted to Phys. Rev. Lett.

[15] Cooper, J. R., Jérome, D., Weger, M. and Etemad, S., J. Physique Lett. 36 (1975) L-219.

[16] Groff, R. P., Suna, A. and Merrifield, R. E., Phys. Rev. Lett. 33 (1974) 418.

Etemad, S., Penney, T., Engler, E. M., Scott, B. A. and SeIDen, P. E., Phys. Rev. Lett. 34 (1975) 741.

[17] Cooper, J. R., Jérome, D., Etemad, S. and Engler, E. M., Solid State Commun. 22 (1977) 257.

[18] Soda, G., Jérome, D., Weger, M., Alizon, J., Gallice, J., Robert, H., Fabre, J. M. and Giral, L., J. Physique 38 (1977) 931.

[19] Berthier, C., CoOper, J. R., Jérome, D., Soda, G., Weyl, C., FABRE, J. M. and Giral, L., Mol. Liq. Cryst. 32 (1976) 267.

[20] JÉrome, D., et al., to be published.

[21] BoufFard, S. and ZuPPIROLI, L., to be published.

[22] Hopfield, J. J., Comments in Solid State Physics 3 (1970) 52.

[23] Mihaly, G., Holczer, K., Janossy, A., Gruner, G. and MILJAK, M., in reference [2].

[24] Pouget, J. P., Khanna, S. K., Denoyer, F., Comès, R., Garito, A. F. and Heeger, A. J., Phys. Rev. 37 (1976) 437 and Phys. Rev. B 16 (1977) 1468.
[25] Kagoshima, S., Ishiguro, T. and Anzai, H., J. Phys. Soc. Japan 41 (1976) 2061.

[26] A similar pressure dependence of the charge transfer in TTF$T C N Q$ has also been found from acoustic attenuation measurements, by J. T. Tiedje, Ph. D. Thesis University of British Columbia 1977.

[27] Welber, B., Engler, E. M., Seiden, P. E. and Grant, P. M., preprint 1976.

[28] Cooper, J. R., Miljak, M., Delplanque, G., Jérome, D., Weger, M., Fabre, J. M. and Giral, L., J. Physique 38 (1977) 1097

[29] Grant, P. M., Greene, R. L., Wrighton, G. C. and CASTro, G., Phys. Rev. Lett. 31 (1973) 1311.

No temperature dependence of $\omega_{\mathrm{p}}$ has been found in the Drude law fit of Bright, A. A., Garito, A. F. and HeEgER, A. J., Phys. Rev. B 10 (1974) 1328.

A blue shift of the plasma frequency $(10 \%)$ at low temperature has also been observed in HMTSF-TCNQ by JACOBSEN, C. S., BeChGAARD, K. and ANDERSEN, J. R., in reference [2].

[30] Torrance, J. B., Tomkiewicz, Y. and Silverman, B. D., Phys. Rev. B 15 (1977) 4738.

[31] Coll, C. F., Phys. Rev. B 9 (1974) 2150.

[32] Emery, V. J., Phys. Rev. Lett. 37 (1976) 107.

[33] Hubbard, J., preprint 1977.

[34] Gorkov, L. P. and DzyaloshinskiI, I. E., JETP Lett. 18 (1974) 401.

[35] The scattering mechanism proposed in the present work conserves the total spin of the electron system and is not therefore a $T_{1}$ relaxation process.

[36] DzyaloshinskiI, J. E. and Larkin, A. I., Sov. Phys. JETP 34 (1972) 422.

[37] Jullien, R., Beal-Monod, M. T. and Copblin, B., Phys. Rev. B 9 (1974) 1441.

[38] We thank G. Grüner for a very helpful discussion on the coupling between collective modes and single particle excitations.

[39] Schultz, A. J., Stucky, G. D., Blessing, R. H., Coppens, P., J. Am. Chem. Soc. 98 (1976) 3194.

[40] Debray, D., Millet, R., Jérome, D., Barišić, S., Giral, L. and FABre, J. M., J. Physique Lett. 38 (1977) L-227. 